\title{
Estimation of above-ground phytomass and carbon in tree resources outside the forest (TROF): A geo-spatial approach
}

\begin{abstract}
B. P. Heyojoo ${ }^{1}$ and S. Nandy ${ }^{2}$
This study aims to estimate above-ground phytomass and carbon of TROF ecosystem in part of Bijnor district in Uttar Pradesh state of India using IRS P6 LISSIV satellite image by geo-spatial approach coupled with field sampling. Chacko's formula was referred to compute number of samples in each TROF types and the sample plot size in each stratum was adopted from Vegetation Carbon Project (VCP) under National Carbon Project (NCP). With the help of field data consisting mainly the height and girth information, volume of each individual tree per plot was obtained using site and tree species-specific standard volumetric equations. The phytomass was calculated by multiplying volume with Biomass Expansion Factor (BEF) then with regional specific gravity of the individual species and summed up in each plot to get total phytomass per plot. The total phytomass per plot was reported to be maximum 544.00 t/ha for linear TROF followed by 121.89 t/ha for block TROF. The carbon from phytomass was obtained by multiplying the total phytomass by a conversion factor that represents the average carbon content in phytomass. Spectral modeling for phytomass with different bands and indices were established and the best fit curve $\left(R^{2}=0.552\right)$ with red band was applied to generate phytomass and carbon distribution map of the study area.
\end{abstract}

Key words: TROF, sampling, geospatial, phytomass, carbon

$\mathrm{G}$ lobal warming has been common topic widely talked everywhere and it is reality. Its impact to all forms of life is inevitable. Scientists have estimated that the average global temperature is likely to rise by 1.4 to $5.8^{\circ} \mathrm{C}$ (UNFCCC, 1998). Increasing green house gases (GHGs) particularly $\mathrm{CO}_{2}$ in atmosphere has alerted scientist community to think over different solutions to lower it. Inter-governmental Panel on Climate Change (IPCC) estimates that the level of $\mathrm{CO}_{2}$ in the atmosphere today is $31 \%$ higher than what it was about 250 years back, at the time of the start of industrial revolution and expected to rise 90 to $250 \%$ over pre-industrial.

The beauty of forest is that it provides significant sink for carbon in addition to providing economic, ecological and socio cultural values. The world's forests and forest soils currently store more carbon than entire atmosphere (FAO, 2006). It is therefore carbon management through forest will probably be the important agenda in the context of GHG effect and mitigation of global climate change. The global initiative of REDD, REDD+ by the United Nation (UN) is collective effort to lower the $\mathrm{CO}_{2}$ by reducing deforestation in developing countries. The Kyoto Protocol to the United Nation Framework Convention on Climate Change (UNFCCC) requires that member countries reduce their human-induced emission of $\mathrm{CO}_{2}$ by at least $5 \%$ below their emission levels of 1990 and allows carbon emission to be balanced by carbon sinks represented by vegetation by 2008-2012 (UNFCCC, 1998).

Although the contribution of tree outside forest (TROF) has been appreciated, little is known about the resources itself (FAO, 2006). In India, TROFs are an important source of wood, other products and environmental services. Such trees include roadside plantings, woodlots, scattered trees in the landscape, trees in fields, home

\footnotetext{
Department of Forest Products and Engineering, Institute of Forestry, Pokhara Campus, Nepal, Email: bheyojoo@hotmail.com

${ }^{2}$ Forestry and Ecology Division, IIRS, Dehradun-248001, India
} 
gardens and orchards. Furthermore, about $80 \%$ of the requirements of the wood-based industries are met from TROFs (Chave et al., 2004). Depending on prevalent land-use patterns and ecological and economic landscape attributes, TROFs also play a role in carbon sequestration, biodiversity conservation, pollution control and erosion control.

TROF is a dynamic resource and its assessment over large areas, in a relatively shorter period, necessitates use of remote sensing (RS) and geographic information system (GIS). The needs for reporting carbon stocks and stock changes under the Kyoto Protocol have placed additional demands for accurate surveying methods that are verifiable, specific in time and space, and that cover large areas at acceptable cost (Dadhwal et al., 2009). So, accurate estimation of TROF biomass is also required for GHG inventories and terrestrial carbon accounting at local to regional scale. In this regard RS especially high spatial resolution satellite imagery has opened effective way to estimate TROF biomass and carbon content.

Phytomass and carbon assessment for TROF have been carried out based on different methodologies for different purpose over last three decades (Adhikari, 2005). Tree pattern in TROF is linear, scatter and block. TROF have relatively low density that makes assessment by conventional methods costly and time-consuming. The very high resolution satellite image is best for TROF assessment, however for large area it is cost prohibitive (Chave et al., 2004). Similarly low resolution satellite image owing to minimum mappable area has scale limitation to accurate TROF mapping. Based on the above discussion, it is imperative to explore different methods on assessment of phytomass and carbon on TROF ecosystem. The present study is one of such attempt using geo-spatial approach to assess the above-ground phytomass and carbon using moderate spatial resolution IRS P6 LISS-IV satellite image coupled with field sampling.

\section{Materials and methods}

\section{Study area}

The study area lies between geo coordinates $29^{\circ}$ $21^{\prime} 00^{\prime \prime}$ to $29^{\circ} 37^{\prime} 00^{\prime \prime} \mathrm{N}$ and $77^{\circ} 59^{\prime} 30^{\prime \prime}$ to $78^{\circ}$ 11'30" E on Bijnor district of UP state of India covering $385 \mathrm{~km}^{2}$ geographical area. The Ganga
River having significantly rich biodiversity and religious importance passes through the western part of the study area flowing north to south as shown in figure 1 . The area, dominantly agrarian, is falling in Tarai Bhabar agro-climatic region and is suitable for large scale production of fruits like mango, guava and papaya, vegetables (tropical and subtropical vegetables) and flowers (gladiolus, roses, tube rose). Nearly $75 \%$ of the people are dependent on it. Of the total area of 38,500 ha, net cultivated area is 33,100 ha forming $86 \%$ of the total area. The area with plain topography has no forest but includes reasonable size of TROF. TROFs are an important source of wood, fuel and fodder, other products and environmental services in the area. Such trees include roadside plantings, woodlots, scattered trees in the landscape, trees in fields, home gardens and orchards. Dalbergia sissoo, Eucalyptus spp, Terminalia arjuna, Mangifera indica, Syzygium cumini, Terminalia rebecca, etc. are major plant species in the study area.

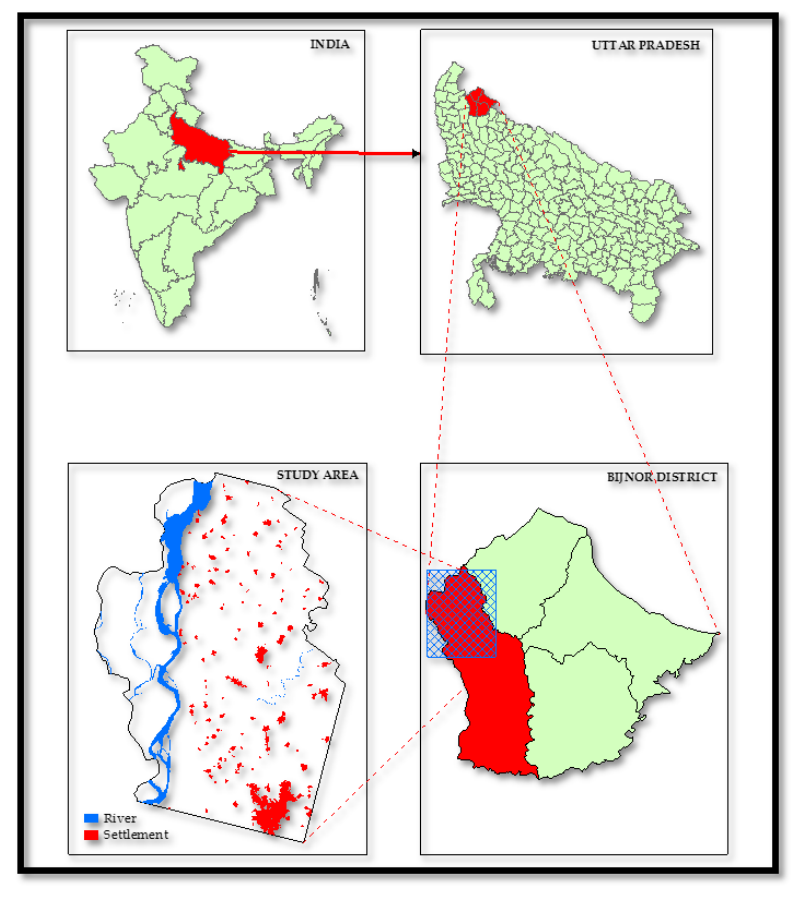

Fig. 1: Study area

\section{Data, tools and software used}

The study utilized remote sensing data, ancillary data and extensive field data.The multi-spectral high spatial resolution data of IRS-P6 LISS-IV sensor was procured from the National Remote Sensing Agency (NRSA), Hyderabad in a digital form (Fig. 2). The IRS-LISS-IV sensor uses a CCD-linear push broom scanning system and provides data in three spectral bands: green, red 


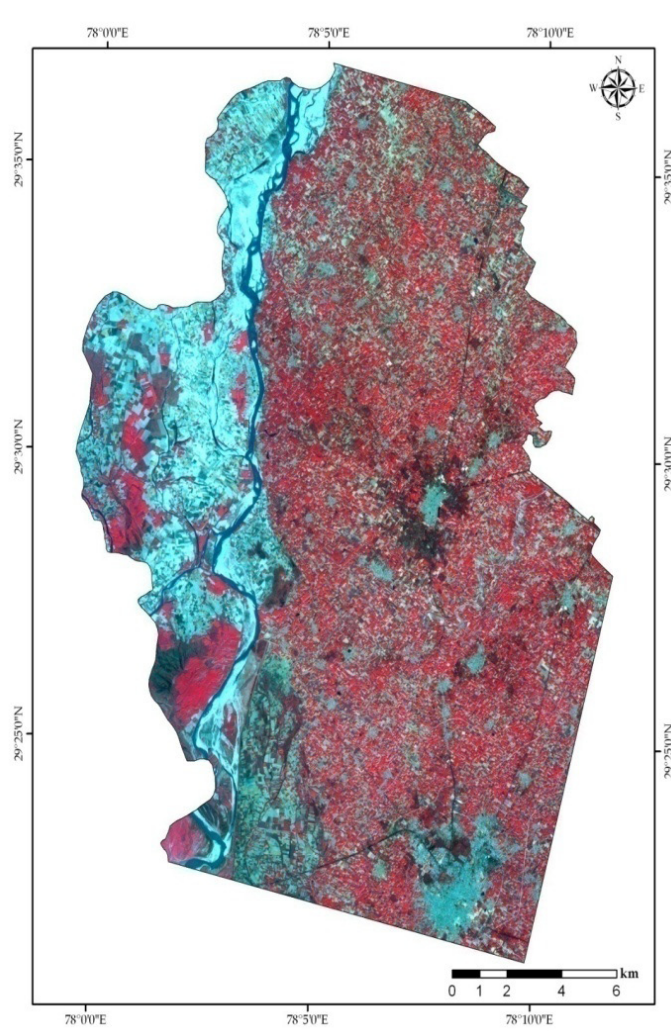

Fig. 2: IRS P6 LISS-IV, 16 March, 2010

and near-infrared with a resolution of $5.8 \mathrm{~m}$. The reason to select the LISS-IV data is mainly due to its high spatial resolution characteristics, which generally portrays more natural representations of objects that assist to map TROF from this data. It can also be used to demarcate small patches of TROF along the agricultural plots easily with the supplement of logical rule and interpretation keys. The present study carried out the analysis of the available single scene of IRS-P6 LISS-IV for the Bijnor district mainly due to limited satellite swath and to avoid the merging of different temporal datasets. Pre-processing and georeferencing of procured satellite data was done in Erdas Imagine 9.2 software where ArcGIS 9.2 was used for creating and merging the spatial and non-spatial database. The well defined and evenly distributed Ground Control Points (GCP) from the field and topo sheets have been used for the geo-referencing of the raw image. It helped to achieve the RMS error of 0.378 , which comes within a pixel. The ancillary datasets such as topographical map (sheet number $53 \mathrm{~K} / 2,53 \mathrm{~K} / 3$, $53 \mathrm{G} / 14$ and $53 \mathrm{G} / 15$ ), Google Image of $0.50 \mathrm{~m}$ spatial resolution of the study area acquired on April 18, 2010 downloaded from the internet using CGUTIL tools were substantially used for visual interpretation. Extensive field work was conducted to collect sampled plot data on species types, number of tress, their diameter and GPS coordinates of the sample plot. Among other secondary data used in the study includes, digital boundary map of the Bijnor District. Historical data of the study area available from state government department website (www.bijnor. nic.in, www.forest.up.nic.in, www.ict.agri.net.in) was also referred in the study.

\section{Research approach}

The whole research was completed through three different steps as pre-field work, field work and post-field work following the conceptual framework (Fig. 3). The pre-field work included preprocessing of satellite data, visual interpretation for TROF classification map. The field work included the sampling inventory for all types of TROF and the post-field work comprised of processing of field data, spectral reflectance analysis for developing relationship with phytomass and satellite parameter.

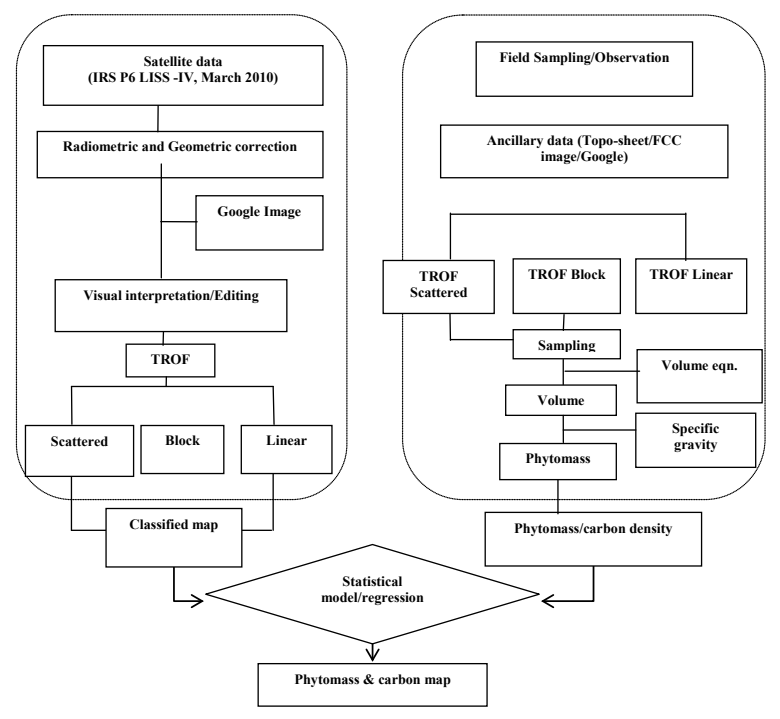

Fig. 3: Conceptual methodological framework

\section{Image rectification, interpretation and TROF classification}

Image to image registration was carried out using georeferenced geotiff image of Landsat ETM + of study area as a master image. Well distributed ground points were chosen throughout the imagery and resampling of the image was done using first order polynomials and nearest neighborhood algorithm in ERDAS Imagine software. Image registration was carried out with UTM $44 \mathrm{~N}$ projection, WGS 84 spheroid and WGS 84 datum. Radiometric correction was applied for radiometric defects and improving 
visual impact of false color composites (FCC). After geometric and radiometric corrections and image to image co-registration, the area of interest (AOI) i.e. part of Bijnor taluk was extracted by overlaying the digitized boundary shape file of the study area. The LISS IV satellite image was visually interpreted to segregate in different landuse/land cover map of the study area. Standard methodology using image key elements such as tone, texture, shape, association, etc were used for visual interpretation in Arc GIS 9.3. Reconnaissance field visit and high resolution satellite images download from Google Earth became great help in visual interpretation for different types of TROF mapping of the area.

\section{Sampling design}

Non-destructive stratified random sampling approach was adopted in different TROF strata produced from the satellite image. A grid of $2 \mathrm{~km} \times 2 \mathrm{~km}$ was overlaid on the topo sheets of the study area. The sampling frames for all types of TROF were defined based on the grids containing particular TROF types under the study. The sample size by Chacko's formula (Chacko, 1964) was computed based on pilot survey measurement of the variance of tree number per plot $(250 \mathrm{~m} \times$ $250 \mathrm{~m}$ ) for six spatially distributed plots. The thirty samples obtained from the formula was adopted for all TROF types and randomly distributed by using random table (Page 18/26, random table by Tippett). All together 90 plots were laid in the field with 30 of $250 \mathrm{~m} \times 250 \mathrm{~m}$ for scattered TROF, 30 of $32 \mathrm{~m} \times 32 \mathrm{~m}$ for block TROF and 30 of $50 \mathrm{~m} \times$ width of linear feature TROF (Fig. 4).

Garmin 12 channel GPS was used for recording coordinates in the middle of all the sampled plots. Detailed data like enumeration of trees, tree species name, average tree height, circumference at breast height $(\mathrm{CBH})$ and altitude were collected from the sample plots.

\section{Organization of field data and phytomass and carbon estimation}

The entire field database was organized in MS Excel. The necessary parameters like volume equation, specific gravity, conversion factors etc. were added in database for further processing. Species and site specific volume equations and specific gravity for respective tree species were noted from literature mainly the publication of FSI (1996) and Forest Research Institute (FRI).
General volume equation of same region was used for tree whose volume equations were not available. The volumes of individual tree were summed up to compute volume per hectare in all types of TROF.

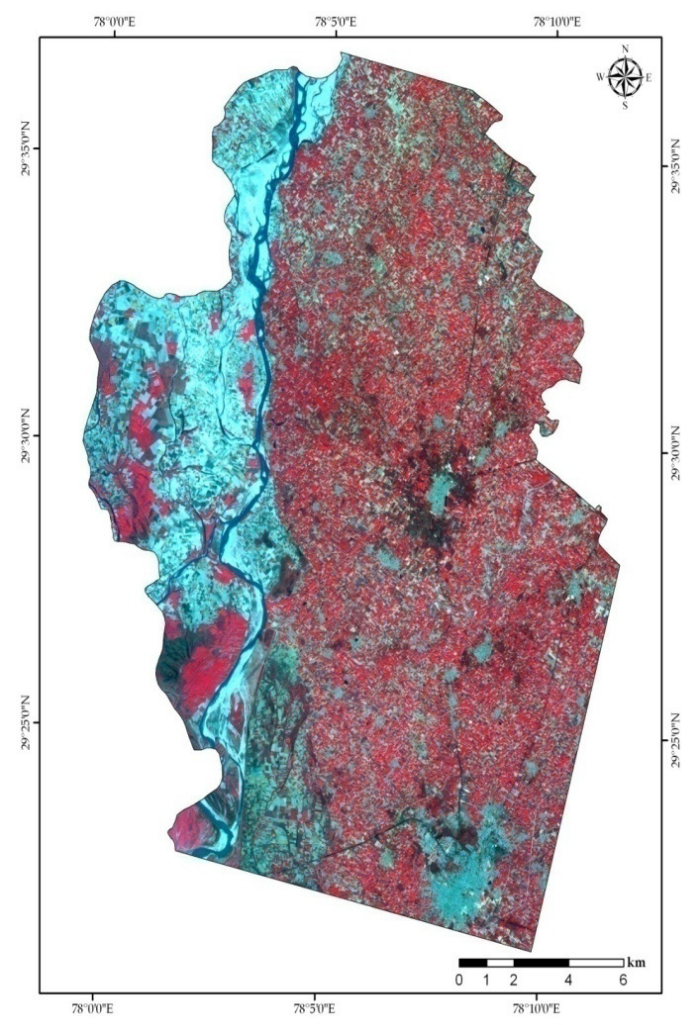

Fig. 4: Sample plot location

\section{Regression analysis and spectral modeling}

The plot wise total phytomass with its spatial location taken by GPS readings during the fieldwork were co-related with different parameters of LISS-IV data such as individual bands pixel value, NDVI, VI to establish best fit equation. Radiometric correction of the image was carried out by dark pixel subtraction method before regression analysis. The regression equation showing highest $\mathrm{R}^{2}$ was used to predict the phytomass and carbon of the study area. About one third of sample data collected in the field was used to test the performance of the best fit regression equation by coefficient of determination $\left(\mathrm{R}^{2}\right)$ and root mean square error (RMSE) calculation.

\section{Results and discussion}

\section{Landuse and land cover map}

The LULC map of the study area included agriculture containing scattered TROF, linear 
TROF, block TROF, settlement, river and water body classes (Fig. 5).The young plantation along linear TROF having crown size less than minimum mappable unit and dry deciduous tree mostly dominated by Populus deltoides could not be mapped due to low spatial resolution and season of the data available.

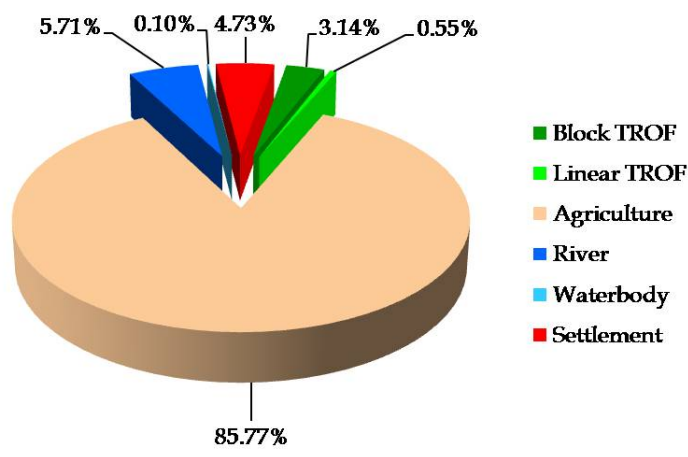

Fig. 5: LULC of the study area

Tree statistics and carbon estimation in different TROF

The study found wide variation in terms of number, diameter size of trees in block and linear types (Table 1) whereas number of trees in scattered was nearly same. The number of trees/ha in linear pattern was varying from 240 to 2680 and that in block plantation was from 160 to 2560 . The diversity of tree species was high in linear than that of block types. The number as well as diameter sizes of tree in linear TROF are high followed by Block TROF. The trees planted along the road side and railways are found to be matured and dense as well. $M$. indica is primarily dominant followed by $E$. spp. in block plantation and E. spp. is dominant followed by mixed species.

The calculations indicates high value in linear followed by block and scattered type (Fig. 6). It is however the total volume for block type is high in the study area followed by linear and scatter types.

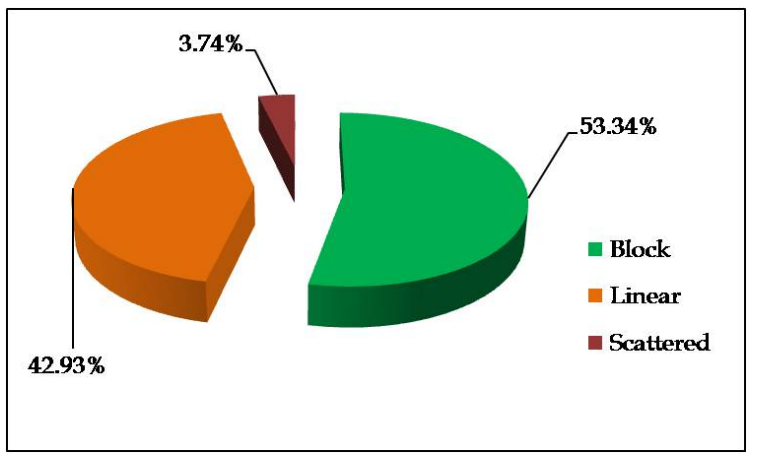

(A)

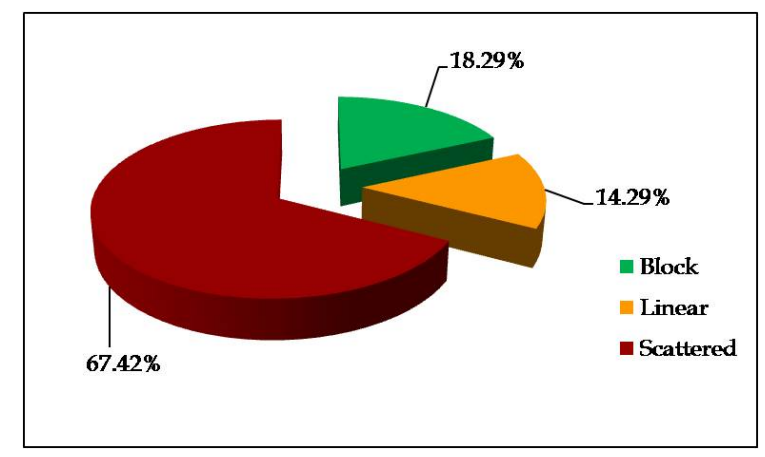

(B)

Fig. 6: Phytomass and carbon per hectare TROF wise (A) Share of total pytomass and carbon in the study area (B)

Phytomass (plant biomass) has direct relationship with amount of carbon present in that plant biomass. Westlake (1963) has observed that there is $47 \%$ carbon present in dry plant biomass, while Intergovernmental IPCC reported that the carbon present in plant biomass is $45 \%$ of it (IPCC, 1995). Based on the results of different studies related to estimation of carbon in wood, it was observed that carbon varies between $45 \%$ to $50 \%$ for different ecosystems and thus $47.00 \%$ carbon has been considered for deriving carbon estimation.

\section{Spectral modeling}

The attempt made in this study showed possibility of using spectral response-based models for biomass estimation. GPS readings taken during the field work at each sample plot were fed into

Table 1: Statistical table of tree and carbon information in different TROF types

\begin{tabular}{lccccrrr}
\hline $\begin{array}{c}\text { TROF } \\
\text { types }\end{array}$ & $\begin{array}{c}\text { Number of } \\
\text { trees/ha }\end{array}$ & $\begin{array}{c}\text { Avg. dia. } \\
(\mathbf{m})\end{array}$ & $\begin{array}{c}\text { Min dia. } \\
\mathbf{( m )}\end{array}$ & $\begin{array}{c}\text { Max dia. } \\
\mathbf{( m )}\end{array}$ & $\begin{array}{c}\text { Average } \\
\text { growing } \\
\text { stock (t/ha) }\end{array}$ & $\begin{array}{c}\text { Average } \\
\text { phytomass } \\
\text { (t/ha) }\end{array}$ & $\begin{array}{c}\text { Average } \\
\text { carbon } \\
\text { (t/ha) }\end{array}$ \\
\hline Block & $160-2560$ & 0.20 & 0.08 & 0.90 & 208.57 & 121.89 & 58.51 \\
Linear & $240-2680$ & 0.34 & 0.10 & 1.21 & 958.84 & 544.00 & 261.12 \\
Scattered & $0-12$ & 0.24 & 0.08 & 1.17 & 0.54 & 16.48 & 7.91 \\
\hline
\end{tabular}


Excel file and later the file was converted into dbf format to generate point shape file. This shape file was overlaid to individual band images, NDVI image, VI image to extract pixel level phytomass. The regression based models with DN value of all individual bands, NDVI, VI were established for $1 \times 1$ window and $5 \times 5$ windows. The various types of equations were tried to find out the best suitable correlation for the given data set i.e. linear, logarithmic, exponential and power. Initially, about $70 \%$ of the sample plots were taken for generating the equations but the corelation was not good. Hence some outlier plots yielding poor co-relation value were removed and the equations were re-established.

The model based on red band with biomass was found to be strongest with the co-relation value $\left(\mathrm{R}^{2}\right)$ of 0.552 (Fig. 7). The phytomass of the whole study area has been calculated by the linear equation as follow;

$$
\begin{aligned}
& y=19.12 x+(-752.9) \\
& m=19.12 \\
& c=-752.9 \\
& x=\text { input red band }
\end{aligned}
$$
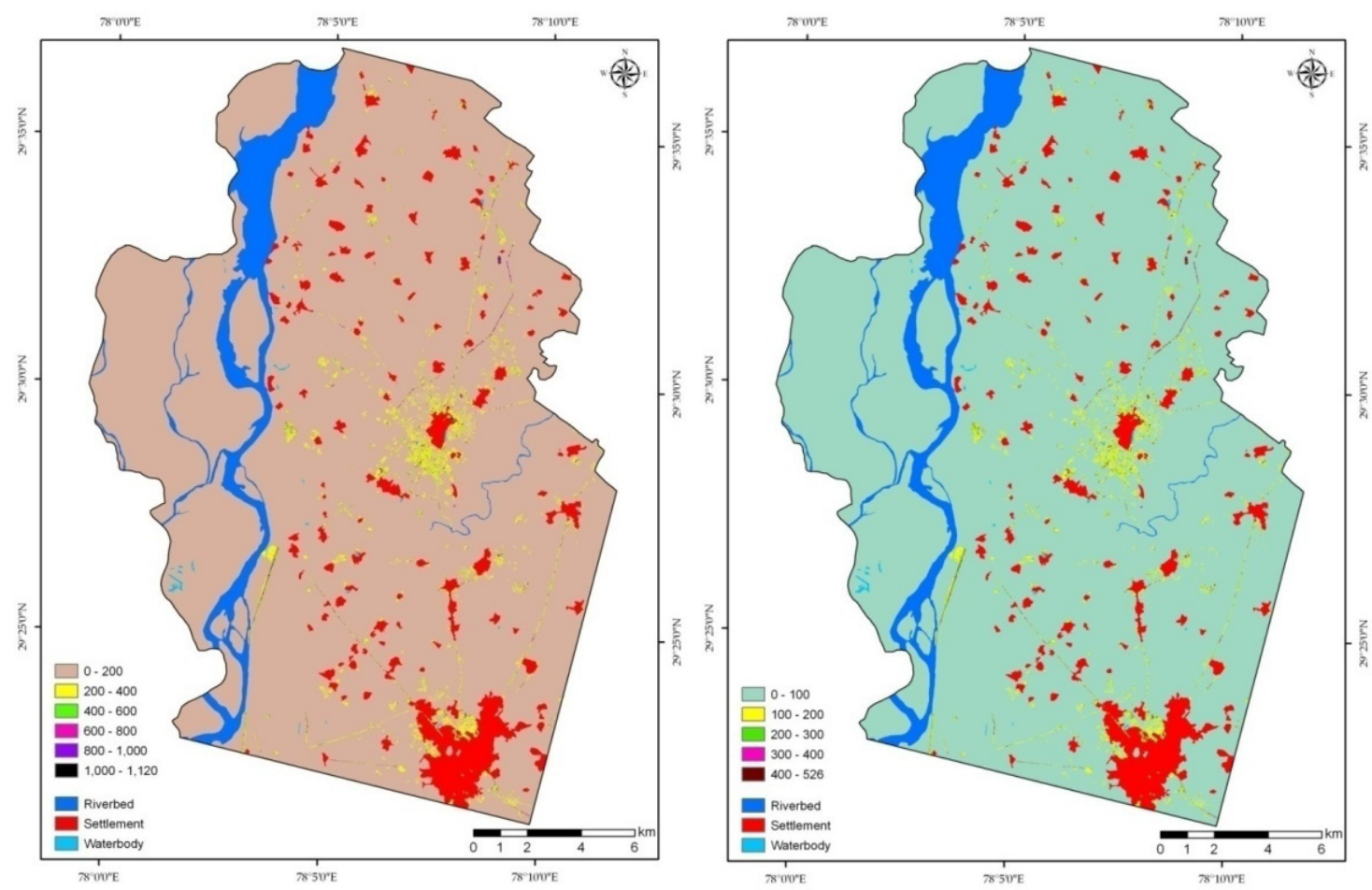

Fig. 8: Phytomass (A) and Carbon (B) distribution map of the study area

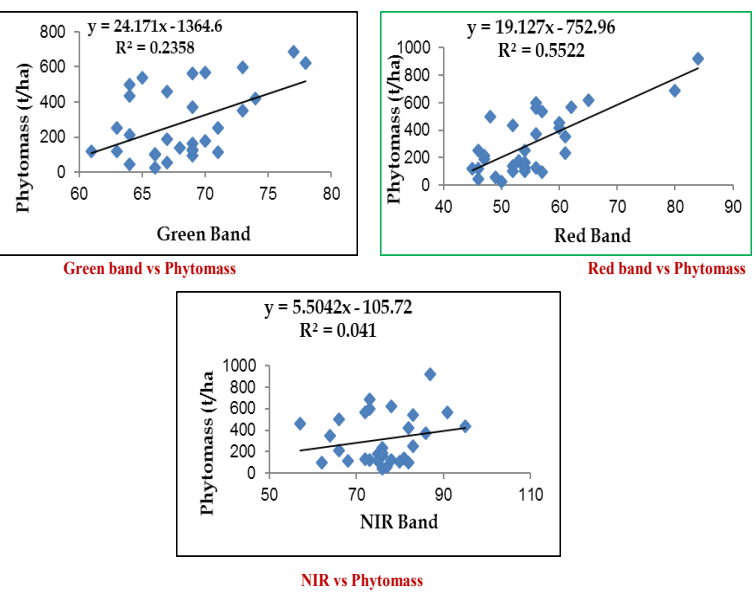

Fig. 7: Regression analysis phytomass with different bands

\section{Phytomass and carbon distribution map}

Based on the best model derived from spectral modeling between red band and biomass, the phytomass (Fig. 8A) and carbon map (Fig. 8B) of linear and block TROF of the study area were generated by masking the riverbed, settlement and waterbody classes. The range of phytomass and carbon in the study area ranges dominantly 200-400 t/ha and 100-200 t/ha respectively. The model has correctly predicted large value of phytomass and carbon along the linear type TROF followed by block type. 


\section{Conclusion}

The roles and impacts of biomass on carbon cycles, soil nutrient allocations, fuel accumulation, and habitat environments in terrestrial ecosystems have long been recognized. The significance of TROF resources particularly like in the study area where no forest exists, becomes high to provide economical, ecological and social service. This study to estimate above ground phytomass and carbon in TROF ecosystem using combined geo-spatial approach and ground sampling using moderate spatial resolution satellite data is one of the few conducted so far. The integration of remote sensing techniques with ground surveys will provide wide area coverage in shorter time spans saving costs. The method can be effectively employed using multi spectral and high-resolution satellite imageries to stratify the TROF resources in such a way that the classification system of TROF resource remains valid. It is however felt that it may not be possible to altogether eliminate ground based data collection looking at information requirements of TROF assessments. It is noteworthy that remote sensing-based AGB estimation is a complex procedure in which many factors such as atmospheric conditions, mixed pixels, data saturation, complex biophysical environments, insufficient sample data, extracted remote sensing variables, and the selected algorithms, may interactively affect AGB estimation.

The empirical models with satellite measured spectral response and biomass indicate that there is a moderate relationship with spectral responses. These relationships have seasonal dependency in varying phonological conditions. The relationship is strongest in red band and poor with vegetation indices. This is attributed to season of data. In addition to that LISS-IV data, having relatively low spectral resolution, has little options to establish robust model as using higher wavelength band like middle infrared bands. This could be more reliable as compared to the visible bands as the later spectral regions are less sensitive to atmospheric changes.

\section{Acknowledgments}

The authors gratefully acknowledge Centre for Space Science and Technology Education in South Asia (CSSTEAP) at Indian Institute of Remote Sensing (IIRS), Dehradun for financial assistance to conduct this study and the organizer of International Conference on Forest, People and Climate at Pokhara in 2013 where constructive feedbacks were received while presenting this paper.

\section{References}

Adhikari, M. 2005. A Non-destructive Approach for Quantitative Assessment of Tree Resources Outside the Forest. M.Sc. Thesis, International Institute for Geo-informatics Science and Earth Observation, Enschede, The Netherlands.

Chave, J., Condit, R., Aguilar, S., Hernandez, A., Lao, S. and Perez, R. 2004. Error propagation and scaling for tropical forest biomass estimates. Philosophical Transactions of the Royal Society of London 359: 409-420.

Dadhwal, V. K., Singh, S. and Patil, P. 2009. Assessment of Phytomass Carbon Pools in Forest Ecosystems in India. NNRMS Bulletin no. 41, 57p.

FAO. 2006. Global Forest Resources Assessment 2005: Progress towards Sustainable Forest Management. FAO Forestry Paper 147. Food and Agriculture Organization of the United Nations, Rome, Italy.

FRI. 1996. Volume Equations for Forester of India, Nepal and Bhutan. Forest Research Institute, Dehradun, Ministry of Environment and Forests, Government of India.

UNFCCC. 1998. 'The Kyoto Protocol to the UNFCCC', in UNFCCC. Report of the Conference of the Parties Third Session, Kyoto, UNFCCC, 4-29. 